\title{
Design and Optimization of Micro-Machined Sierpinski Carpet Fractal Antenna Using Ant Lion Optimization
}

\author{
Ashish Kumar ${ }^{*}$, Amar Partap Singh Pharwaha \\ Department of Electronics and Communication Engineering, Sant Longowal Institute of Engineering and Technology, \\ Longowal-148106, Sangrur, Punjab, India
}

Received 29 April 2020; received in revised form 27 July 2020; accepted 25 September 2020

DOI: https://doi.org/10.46604/ijeti.2020.5596

\begin{abstract}
This study investigates the optimized Sierpinski carpet fractal patch antenna and also explores the possibility of the integration of the proposed design with monolithic microwave integrated circuits. The optimization process has been performed using an ant lion optimization algorithm to achieve the required operating frequency and impedance matching. Further, due to surface waves excitation in the high index substrates used for the antenna design, the performance of the antenna degrades. Therefore, a process of micro-machining has been adopted to overcome this limitation. The micro-machining process creates an air cavity underneath the patch which further creates the low index environment in the patch antenna causing drastic improvement in the performance parameters along with the compatibility with monolithic microwave integrated circuits. The design shows multiple resonance frequencies in $\mathrm{X}$-band and Ku-band. The proposed micro-machined design shows the resonance at $7.9 \mathrm{GHz}, 9.6 \mathrm{GHz}, 13.6 \mathrm{GHz}$, and $19 \mathrm{GHz}$ with a maximum gain of $6 \mathrm{dBi}$.
\end{abstract}

Keywords: ant lion optimization, gain, bandwidth, micro-machining, fractal, high index substrate

\section{Introduction}

Investigations on multiband and high gain antennas have attracted attention in the last decade because of the evolution of various wireless communication applications operating from few $\mathrm{MHz}$ to several GHz. Above mentioned characteristics can be achieved with the utilization of microstrip patch antenna into the system due to its various advantages over the conventional antennas [1-3].

Among those properties, the multiband property of the patch antenna can be achieved by transforming the conventional patch structure into the fractal geometry [4-5]. There are various conventional and modified fractal geometries having been discussed in the literature to design the patch antenna. Among them, some are very popular named as Sierpinski carpet, Sierpinski gasket, Hilbert curve, Minkowski curve, Koch fractal, tree fractal etc. [6-13].

From the past decade, other hybrids and modified fractal structures have also been introduced in the literature to design the patch antenna and correspondingly improve the performance parameters. This study investigates the optimized version of the Sierpinski carpet fractal antenna up to the third iteration.

The performance parameters mainly depend upon the dimensional configuration of the design; however, most of the time, it is a very tedious task for the antenna designers to achieve the required band of operation with the exact calculated

\footnotetext{
* Corresponding author. E-mail address: ashish@sliet.ac.in

Tel.: +91-9815734458
} 
dimensions of the patch antenna. Therefore, dimensions of the proposed antenna have to be optimized to achieve the required performance characteristics; for optimization, several evolutionary algorithms have been presented for solving the various single objective and multi-objective functions [14].

Regarding the microstrip patch antenna, several parameters like dimensions of the patch and ground plane, feed position, width of microstrip feed, and height of the substrate can be optimized to attain the expected resonant frequency, bandwidth, and return loss and gain [15]. Various algorithms like particle swam optimization (PSO), bacterial foraging optimization (BFO), whale optimization, grey wolf optimization, ant lion optimization (ALO), etc. have been used to optimize the dimensions of the antenna and to achieve the required results [16-17]. In this study, the ant lion optimization has been applied on the length of the patch and width of the quarter wave matching line to obtain the required band of operation and corresponding reflection coefficient, respectively.

From the theoretical and practical point of view regarding patch antenna, it is evident that the choice of substrate material is the crucial step toward determining the performance parameters of the antenna. Generally, thick and low dielectric material substrates like roger (2.2) and FR4 (4.4) are used to achieve better performance. But to design, the antenna with small size and compatible with monolithic microwave integrated circuits (MMIC), high index materials like silicon (11.9) and Gallium Arsenide (12.9) are preferred.

Traditionally, there is a drawback of using high index materials directly due to the generation of surface waves which further degrades the performance of the patch antenna [18]. To deal with the situation, a selective part of the substrate materials will be etched off underneath the substrate material with the process called bulk micro-machining [19], which further reduces the overall dielectric constant of the substrate and improves the performance of the proposed antenna.

The idea of micro-machining comes from drilling the holes (like EBG) in conventional substrates like FR4 or Duroid to improve the antenna performance characteristics [20].There is another type of micro-machining called surface micromachining which is utilized in the designing of RF-MEMS switches of cantilever type beam and shunt capacitive type beams. In this paper, the bulk micro-machining of the final iteration of the design has been performed and performance parameters have been improved.

\section{Proposed Antenna Design}

This section consists of the proposed antenna design and divides into two subsections, one of which includes a conventional Sierpinski carpet fractal patch antenna designed on FR4 substrate and another shows the utilization of micromachining in the $3^{\text {rd }}$ iteration of the design. The proposed design has been iterative up to the $3^{\text {rd }}$ iteration. All the designs have been simulated on high-frequency structure simulator (HFSS 13.0) software.

\subsection{Conventional Sierpinski fractal antenna}

To design the proposed fractal design, dimensions of the rectangular patch have been calculated using standard equations to design Zeroth iteration of the Sierpinski carpet antenna on substrate of thickness $1.58 \mathrm{~mm}$ with dielectric constant 4.4 (FR4) and loss tangent of 0.02. The first iteration is formed by cutting a slot in the center having one-third dimensions of the patch in Zeroth iteration. The second iteration is formed by cutting the 9 slots around the center slot by calculating one-ninth dimensions of the Zeroth iteration and the process continues as shown in Fig. 1.

However, more mathematical approaches like exact numerical results instead of limits and to calculate exact infinitesimal values of the area can be observed from [21-23]. Further, the dimensional view of the $3^{\text {rd }}$ iteration is shown in Fig. 2 and various nomenclatures regarding dimensions have been tabulated in Table 1 . A microstrip feed of $50 \Omega$ is used with a $\lambda / 4$ feed line to achieve the matching with the patch antenna. 


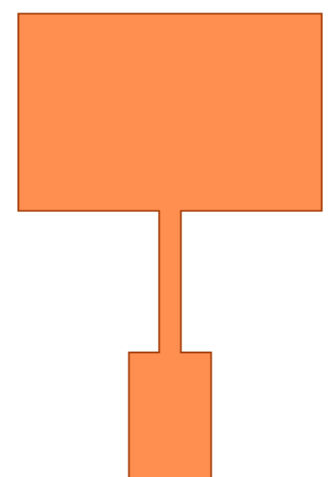

(a) Zerothiteraiton

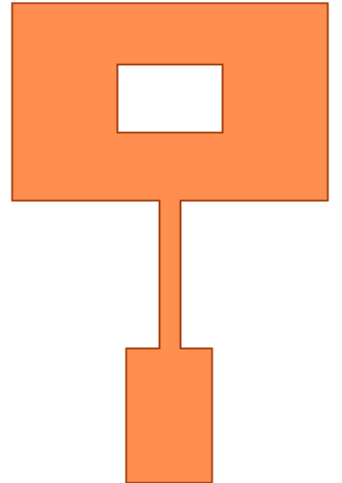

(b) First iteration

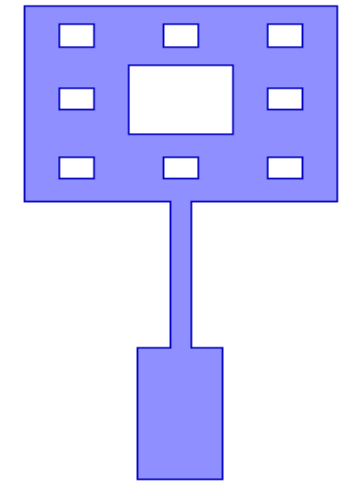

(c) Second iteration

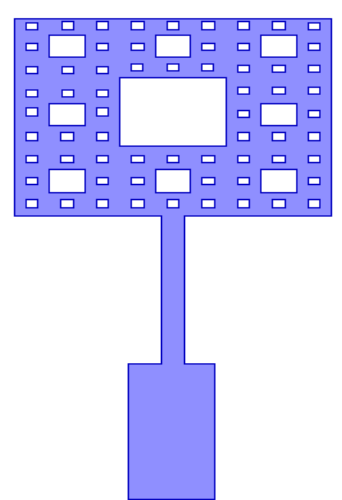

(d) Third iteration

Fig. 1 Sierpinskicarpet fractal patch antenna iterations

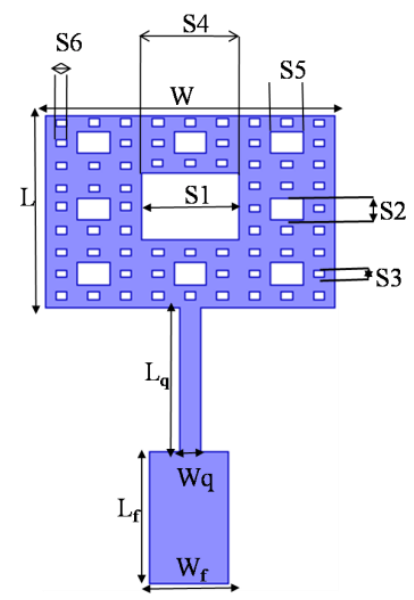

Fig. 2 Dimensional view of the third iteration of the proposed design

Table 1 Dimensional parameters of the proposed antenna

\begin{tabular}{|c|c|}
\hline Parameters & Dimension (in mm) \\
\hline Length of the patch (L) & 7.08 \\
\hline Width of the Patch (W) & 10.89 \\
\hline Width of Slot 1 (S1) & 3.63 \\
\hline Width of Slot 2 (S2) & 1.21 \\
\hline Width of Slot 3 (S3) & 0.4 \\
\hline Length of Slot 1 (S4) & 2.49 \\
\hline Length of Slot 2 (S5) & 0.79 \\
\hline Length of Slot 3 (S6) & 0.26 \\
\hline Length of quarter wave matching line $\left(\mathrm{L}_{\mathrm{q}}\right)$ & 5.3 \\
\hline Width of quarter wave matching line $\left(\mathrm{W}_{\mathrm{q}}\right)$ & 0.75 \\
\hline Width of Feed $\left(\mathrm{W}_{\mathrm{f}}\right)$ & 2.95 \\
\hline Length of feed $\left(\mathrm{L}_{\mathrm{f}}\right)$ & 4.85 \\
\hline
\end{tabular}

To do so, first, the impedance of the patch has been calculated which comes around $300 \Omega$ and to match this impedance with the $50 \Omega$, impedance a quarter wave matching line is connected. The impedance of the quarter wave line was calculated as $Z_{q}=\sqrt{300 \times 50}=122.47 \Omega$. Further, the width of the microstrip line has been decided by the impedance of the microstrip line. Therefore, from the calculated impedance of the quarter wave matching line, the corresponding width of the same has been found using an online microstrip calculator.

The Sierpinski carpet is constructed using squares geometry of the patch antenna. After calculating all the dimensional parameters, the next step is to study the mathematics of the fractal geometry in which let $N_{n}$ be the number of yellow boxes of the patch, $L_{n}$ is the ratio for the length, $A_{n}$ is the ratio of the fractional area after the nth iteration and $d_{n}$ is the capacity dimension [24]. Then: 


$$
\begin{aligned}
& N_{n}=8^{n} \\
& L_{n}=\left(\frac{1}{3}\right)^{n} \\
& A_{n}=\left(\frac{8}{9}\right)^{n} \\
& d_{n}=-\lim _{n \rightarrow \infty}\left(\frac{\ln N n}{\ln L n}\right)=1.89
\end{aligned}
$$

\subsection{Micro-machined Sierpinski fractal antenna}

The performance of the $3^{\text {rd }}$ iteration of the proposed design has been improved using the process called micromachining as shown in Fig. 3. In this process, a selective part of the substrate material etched off underneath the patch while remaining the other circuitry on the substrate. Conventionally, the micro-machining process has been implemented on the high index substrate. But due to high surface wave excitation in high index materials, the performance parameters (gain and operational bandwidth) of the antenna degrade.

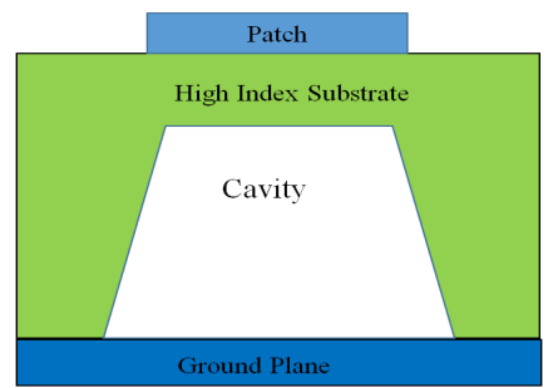

Fig. 3 Micro-machining in antenna

Consequently, a low index environment has been created underneath the patch antenna by creating a cavity; this process will improve the performance parameters of the patch antenna [18]. The overall effective dielectric constant after the micromachining process has been calculated using Eqs. (5)-(8).There is a region of the mixed air-silicon. The effective dielectric constant can be calculated by using the cavity model. The capacitance of the patch in mixed region is given by:

$$
C=\frac{\varepsilon_{e f f} A}{d}
$$

where $\mathrm{C}$ is the capacitance, $\mathrm{A}$ is the area of the patch, $\mathrm{d}$ is the substrate thickness and $\varepsilon_{e f f}=\varepsilon_{e f f} \varepsilon_{0}$. In this context, walls of the cavity are assumed to be straight and $\varepsilon_{\text {reff }}$ can be estimated from the following equations [18]:

$$
\begin{gathered}
\varepsilon_{\text {cavity }}=\frac{\varepsilon_{\text {air }} \varepsilon_{\text {sub }}}{\varepsilon_{\text {air }}+\left(\varepsilon_{\text {sub }}-\varepsilon_{\text {air }}\right) X_{\text {air }}} \\
\frac{\varepsilon_{\text {fringe }}}{\varepsilon_{\text {cavity }}}=\frac{\varepsilon_{\text {air }}+\left(\varepsilon_{\text {sub }}-\varepsilon_{\text {air }}\right) X_{\text {air }}}{\varepsilon_{\text {air }}+\left(\varepsilon_{\text {sub }}-\varepsilon_{\text {air }}\right) X_{\text {fringe }}} \\
\varepsilon_{\text {reff }}=\varepsilon_{\text {cavity }}\left(\frac{L+2 \Delta L \frac{\varepsilon_{\text {fringe }}}{\varepsilon_{\text {cavity }}}}{L+2 \Delta L}\right)
\end{gathered}
$$


In the above formula, $\varepsilon_{s u b}$ is the dielectric constant of the substrate, $\varepsilon_{\text {air }}$ is the dielectric constant of air, $\mathrm{X}_{\text {air }}$ is the ratio of the air to full substrate thickness in the mixed field region, $\mathrm{L}$ is the length of patch and $\mathrm{X}_{\text {fringe }}$ is ratio of the air to full substrate thickness in the fringing field regions. The top view and 3D view of the micro-machined Sierpinski carpet fractal patch antenna have been shown in Figs. 4-5, respectively.

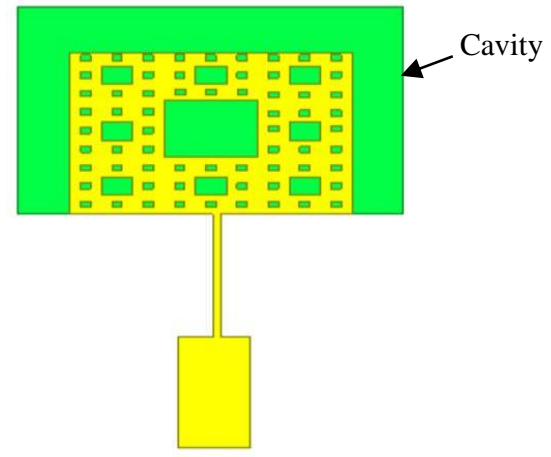

Fig. 4 Top view of the micro-machined Sierpinski carpet antenna

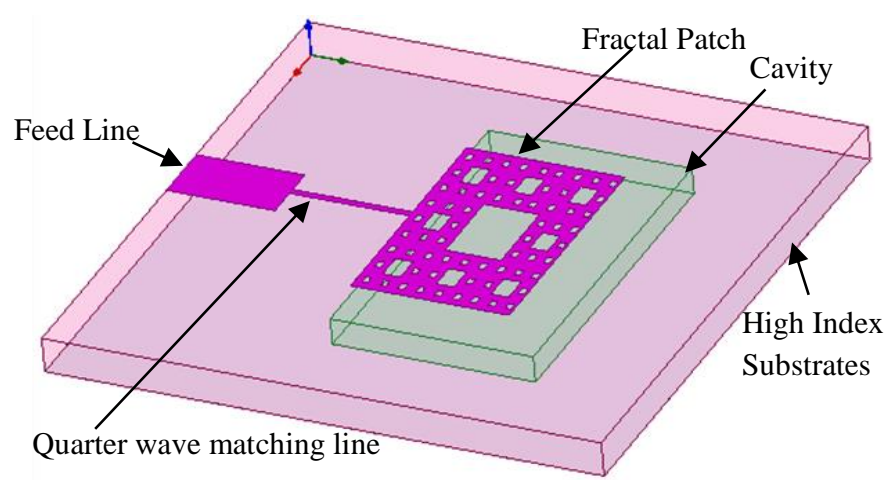

Fig. 5 3D view of the micro-machined Sierpinski carpet antenna

\section{Optimization of the Sierpinski Carpet fractal Patch Antenna}

This section comprises of two sections namely curve fitting implementation and ant lion optimization algorithm. The length of the patch and width of quarter wave matching line have been optimized to achieve the required band of operation and reflection coefficient.

\subsection{Curve fitting implementation}

The curve fitting tool in matlab is a method to establish relations between input and output parameters. The relations are shown in the form of polynomial equations from $2^{\text {nd }}$ order to several order equations. Also, the curve fitting shows the residuals and least mean square error methods. Residual shows that how much the calculated data will be deviated from the actual fitting curve [25].

In the antenna design, curve fitting is used to correlate the dimensions of the patch antenna and performance parameters. In this paper, a relation has been established between the length of the patch and resonant frequency as the resonant frequency of the antenna is inversely proportional to the length of the patch. Similarly, return loss depends on the width of the quarter wave transformer, a relation has been setup between return loss and quarter wave transformer. A set of values has been tabulated from the simulation results.

\subsection{ALO implementation}

The name antlion comes from their distinctive nature of hunting of their prey. Antlion larvae excavates a sharp -edged cone like a hole where it waits for the prey. The prey like an ant can slip into the cone-shaped hole. The prey tries to rescue from the hole. But the predator captures the prey by throwing sand onto the prey and consumes the body of ant. After consuming the ant, antlions throw the remaining parts of the prey and build the pit for the next hunt. For imitating this process, it is supposed that ant would be caught when ant's fitness is more than its equivalent antlion. An antlion updates its position to catch other ants. This process is imitated with Eq. (9) [26]:

$$
\text { Antlion }_{k}^{i}=\operatorname{Ant}_{k}^{i} \text { if fitness }\left(\text { Ant }_{k}^{i}\right)>\operatorname{fitness}\left(\text { Antlion }_{k}^{i}\right)
$$

Here $A n t l i o n_{k}^{i}$ is the position of $k^{\text {th }}$ antlion in $i^{\text {th }}$ iteration and $A n t_{k}^{i}$ shows the position of $k^{\text {th }}$ ant at $i^{\text {th }}$ iteration, Elitism gave the fittest antlion produced so far in all iterations and known as elite. Because of this, the movement of all the ants will 
be affected by the elite. Hence, every ant updates their position according to Eq. (10) [26] All the input parameters of the ant lion optimization algorithm related to the design are shown in Table 2.

$$
\text { Ant }=(\text { Roulette wheel selection+random walk selection }) / 2
$$

The pseudo codes for the ALO algorithm are defined as follows [26]:

Initialize the first population of ants and antlions randomly

Calculate the fitness of the ants and antlions

Find the best antlions and assume it as the elite

While the end criterion is not satisfied

for every ant

Select an antlion using Roulette wheel

update ant positions by shrinking the radius

Create a random walk and normalize it

end for

Update the positions of ant using equation (3.7)

Calculate the fitness of all ants

Replace an antlion with its corresponding ant it if becomes fitter (3.6)

Update elite if an antlion becomes fitter than the elite

end while

Return elite

Table 2 Input parameters of ALO

\begin{tabular}{|c|c|c|}
\hline S.No. & Details of Parameter & Values \\
\hline 1 & Number of Search Agents & 100 \\
\hline 2 & Lower Bound for L & $5 \mathrm{~mm}$ \\
\hline 3 & Upper Bound for L & $9 \mathrm{~mm}$ \\
\hline 4 & Lower Bound for Wq & $0.1 \mathrm{~mm}$ \\
\hline 5 & Upper Bound for Wq & $1 \mathrm{~mm}$ \\
\hline 6 & Dimensions (number of variables) & 2 \\
\hline 7 & Max Iterations & 100 \\
\hline
\end{tabular}

\section{Results and Discussions}

This section discusses the various results regarding the above designs and optimization. At first, optimized results have been demonstrated to achieve the required operating frequency with excellent impedance matching. After that overall result of all the iterations has been discussed and the finally, the effect of micro-machining on the third iteration has been discussed.

\subsection{Analysis of conventional and optimized Sierpinski carpet patch antenna}

The calculated length of the patch is $7.95 \mathrm{~mm}$. At this length, the resonant frequency is $7.9 \mathrm{GHz}$. But the required frequency is $8.3 \mathrm{GHz}$. So to obtain this frequency, the length of the patch is optimized with the fitness function given in Eq. (11). The fitness function is minimized using ant lion optimizer. Further, Eq. (12) has been formulated using a curve fitting technique by establishing a relation between the length of the patch and the operating frequency.

$$
\begin{aligned}
& \text { Fitness Function }=(8.3-F)^{2} \\
& F=-0.12785 \times L^{5}+4.5376 \times L^{4}-64.16 \times L^{3}+452.14 \times L^{2}-1588.9 \times L+2239.2
\end{aligned}
$$

The resonant characteristics of the conventional Sierpinski carpet operate at different frequencies in X-band. To improve bandwidth and to obtain the required frequency band length of the patch have to be optimized by using Eqs. (11) and (12) through the ant lion optimizer. After applying the optimization obtained value of the patch length is $7.08 \mathrm{~mm}$ which is less than the actual calculated length. So, the overall area of the patch antenna has been miniaturized. At the optimized 


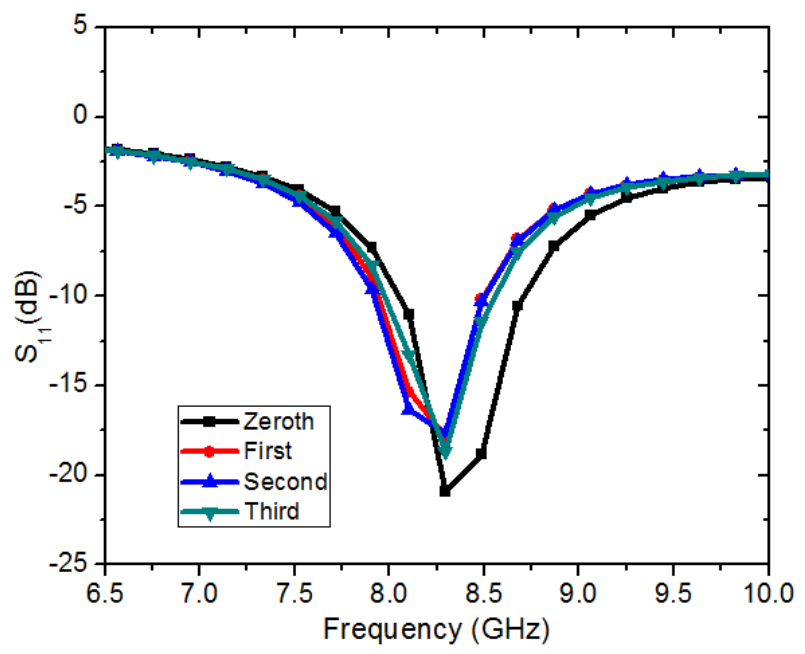

Fig. 6 Optimized resonant frequency

length, the resonance characteristics of all the iterations for X-band are shown in Fig. 6, which illustrates that the resonant frequency for all the iterations is $8.3 \mathrm{GHz}$ and all the iterations cover the 8025 to $8400 \mathrm{MHz}$ band.

\subsection{Analysis of Sierpinski carpet fractal antenna with optimization of width of quarter wave transformer}

In this section, the effect of the width of the quarter wave transformer on the return loss has been discussed. For the third iteration, the variation in the return loss with width of quarter wave transformer at $8.3 \mathrm{GHz}$ has been formulated using curve fitting approach which is described in:

$$
R L=397.62 \times\left(W q^{5}\right)-1478.1 \times\left(W q^{4}\right)+2004.2 \times\left(W q^{3}\right)-1104 \times\left(W q^{2}\right)+206.24 \times W q-24.1
$$

Eq. (13) is formulated to get the best return loss and also to obtain the optimized value of the width of the quarter wave transformer. The fitness function has been established to find the best return loss at optimized feed width, which is given in:

$$
\text { Fitness function }=(26+R L)^{2}
$$

The above fitness has been optimized through ant lion optimizer. Accordingly, the best return loss has been found to be $-26 \mathrm{~dB}$ which is better than the conventional return loss $(-18 \mathrm{~dB})$ and optimized width of the quarter wave transformer is $0.5797 \mathrm{~mm}$, which is also less than the calculated width i.e. $0.7 \mathrm{~mm}$. The results of the optimized and conventional return loss are shown in Fig. 7. Basically, higher return loss shows better impedance matching and subsequently, more power is radiated into space with low loss of power, which will significantly improves the efficiency of the antenna.

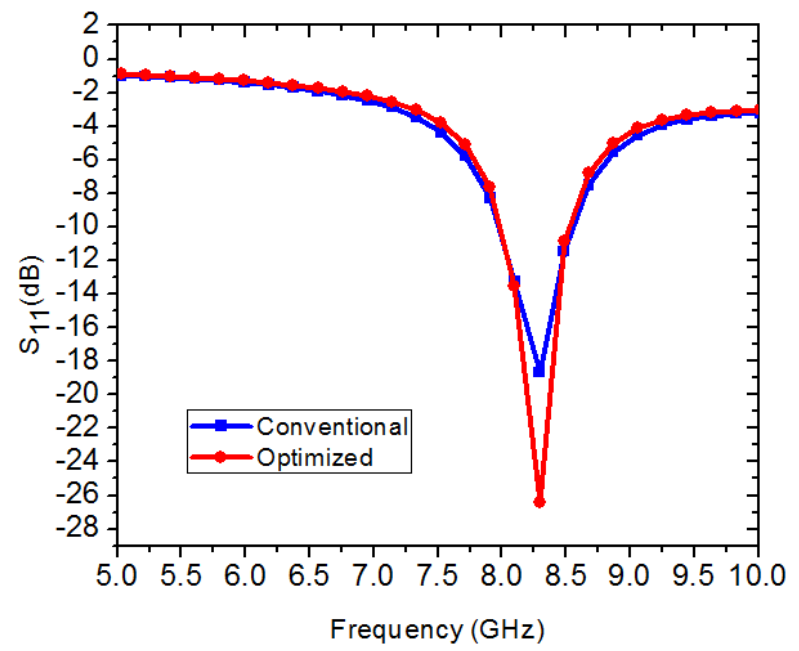

Fig. 7 Optimized reflection coefficient 


\subsection{Overall analysis of optimized Sierpinski carpet fractal antenna}

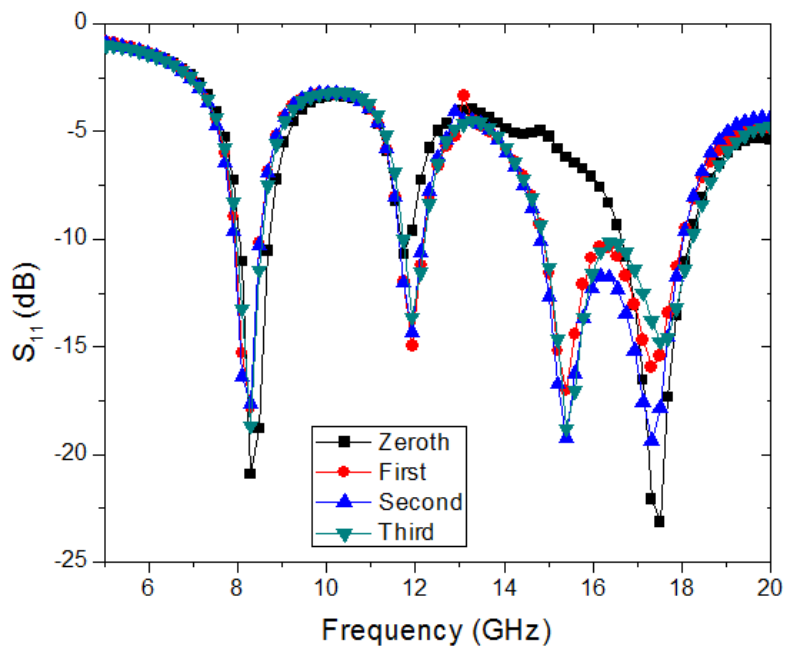

Fig. 8 Frequency v/s $S_{11}(d B)$ curve of Sierpinski carpet fractal antenna

Table 3 Performance parameters of the optimized Sierpinski carpet fractal antenna

\begin{tabular}{|c|c|c|c|c|}
\hline \multicolumn{5}{|c|}{ Zeroth Iteration } \\
\hline Resonant Frequency & $\mathrm{S}_{11}(\mathrm{~dB})$ & Gain $(\mathrm{dBi})$ & Bandwidth & VSWR \\
\hline 8.2929 & -20.91 & 3.15 & $660 \mathrm{MHz}$ & 1.1977 \\
\hline 17.5051 & -23.15 & 8.67 & $1.567 \mathrm{GHz}$ & 1.1495 \\
\hline \multicolumn{5}{|c|}{ First iteration } \\
\hline 8.2929 & -17.84 & $2.6 \mathrm{~dB}$ & $555 \mathrm{MHz}$ & 1.2941 \\
\hline 11.9394 & -14.95 & $2.8 \mathrm{~dB}$ & $558 \mathrm{MHz}$ & 1.4355 \\
\hline 15.3939 & -17.05 & 6.84 & $3.14 \mathrm{GHz}$ & 1.3267 \\
\hline \multicolumn{5}{|c|}{ Second Iteration } \\
\hline 8.2929 & -17.64 & 4.5 & $581 \mathrm{MHz}$ & 1.3017 \\
\hline 11.9394 & -14.32 & 2.7 & $522 \mathrm{MHZ}$ & 1.4761 \\
\hline 17.3131 & -19.37 & 9.26 & $3.226 \mathrm{GHz}$ & 1.2408 \\
\hline \multicolumn{5}{|c|}{ Third Iteration } \\
\hline 8.2929 & -18.68 & 2.5 & $580 \mathrm{MHz}$ & 1.2634 \\
\hline 11.9394 & -13.62 & 2.85 & $477.6 \mathrm{MHz}$ & 1.5263 \\
\hline 15.3939 & -18.80 & 5.97 & $3.36 \mathrm{GHz}$ & 1.2593 \\
\hline \multicolumn{5}{|c|}{}
\end{tabular}

The bandwidth of all the bands has been improved with the optimization process, which is shown in Fig. 8 and Table 3 . Fig. 8 illustrates the resonance characteristics of the optimized Sierpinski carpet fractal antenna operating in X-band and KuBand. From Table 3, it can be seen that all the iterations in Sierpinski carpet fractal antenna resonant at desired frequency which is $8.3 \mathrm{GHz}$. It can also be observed that one more frequency about $11.9 \mathrm{GHz}$ has been achieved which is at the edge of the $\mathrm{X}$-band and the rest of the frequencies belongs to the Ku-band with large bandwidth.

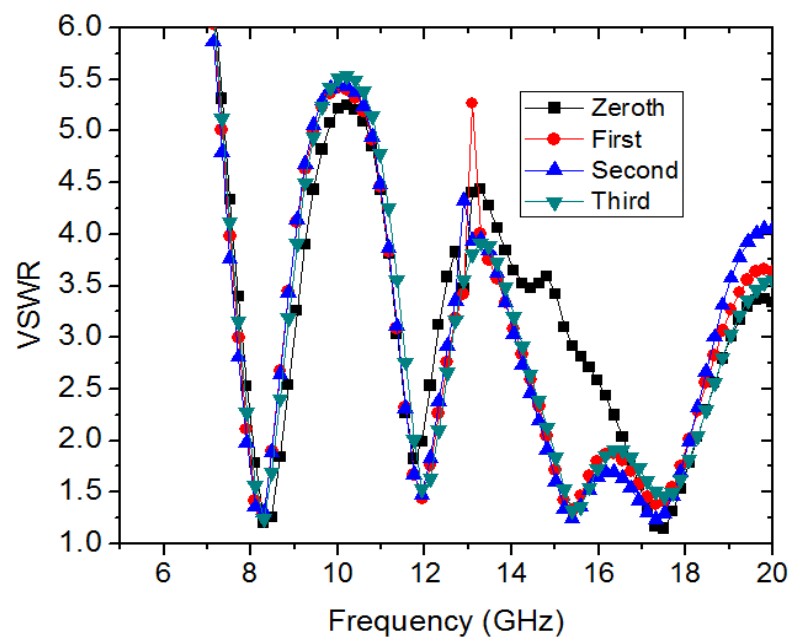

Fig. 9 Frequency v/s VSWR curve of Sierpinski carpet fractal antenna 
Moreover, VSWR graph has been shown in Fig. 9, which depicts that the value of VSWR is less than 2 at the respective operating frequencies. VSWR can also be calculated from the return loss and reflection coefficient and vice versa from:

$$
T=\frac{V S W R-1}{V S W R-1}
$$

where $\mathrm{T}$ is the reflection coefficient

$$
R L=-20 \log _{10}(\mathrm{~T})
$$

\subsection{Radiation patterns of the conventional Sierpinski carpet fractal antenna design}

The radiation pattern consisting of co-polarization and cross-polarization at all the resonating frequencies of the $3^{\text {rd }}$ iteration of the proposed design has been shown in Fig. 10. There are two radiations patterns for each operating frequency i.e., one at $\mathrm{phi}=0^{\circ}$ and another at $\mathrm{phi}=90^{\circ}$.

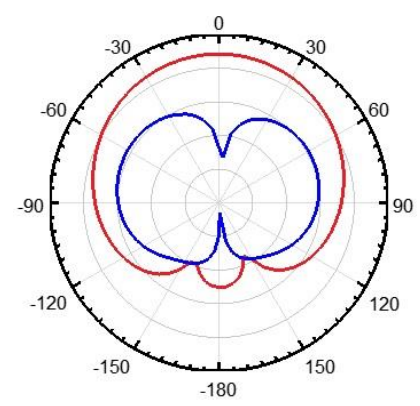

(a) $\phi=0^{\circ}$ and $\mathrm{f}=8.29 \mathrm{GHz}$

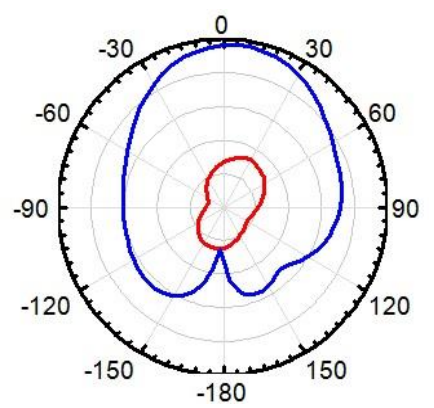

(d) $\phi=90^{\circ}$ and $\mathrm{f}=11.93 \mathrm{GHz}$

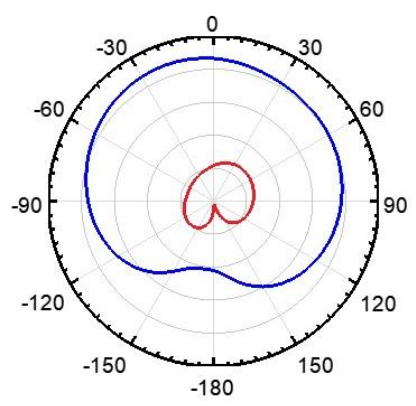

(b) $\phi=90^{\circ}$ and $\mathrm{f}=8.29 \mathrm{GHz}$

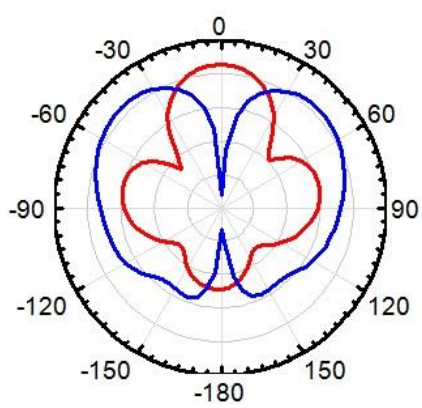

(e) $\phi=0^{\circ}$ and $\mathrm{f}=15.39 \mathrm{GHz}$

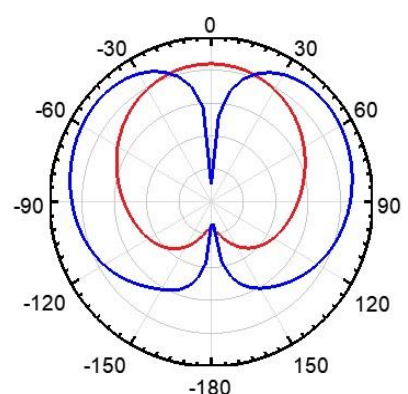

(c) $\phi=0^{\circ}$ and $\mathrm{f}=11.93 \mathrm{GHz}$

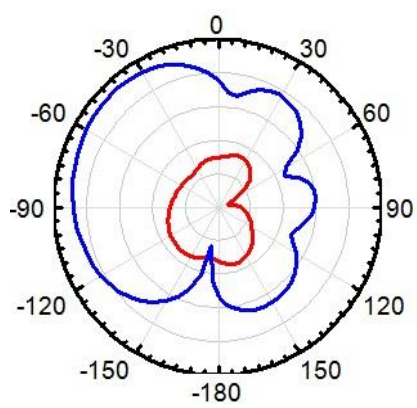

(f) $\phi=90^{\circ}$ and $\mathrm{f}=15.39 \mathrm{GHz}$

Fig. 10 Simulated co and cross polarization of the antenna

It can be observed that the good cross-polarization level lower than $30 \mathrm{~dB}$ in the direction perpendicular to the antenna with a maximum gain of $5.8 \mathrm{~dB}$ is observed in the third band. The $3 \mathrm{D}$ gain patterns at the respective operating frequencies are shown in Fig. 11. Various performance parameters like resonating frequency, $S_{11}(\mathrm{~dB})$, VSWR, and gain of all the iterations have been shown in Table 3.

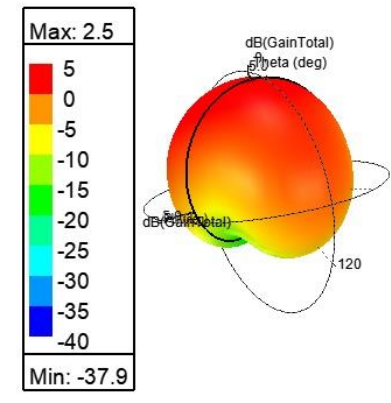

(a) Gain plot at $8.29 \mathrm{GHz}$
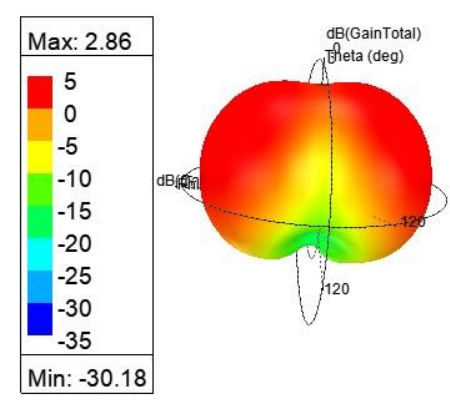

(b) Gain plot at $11.93 \mathrm{GHz}$

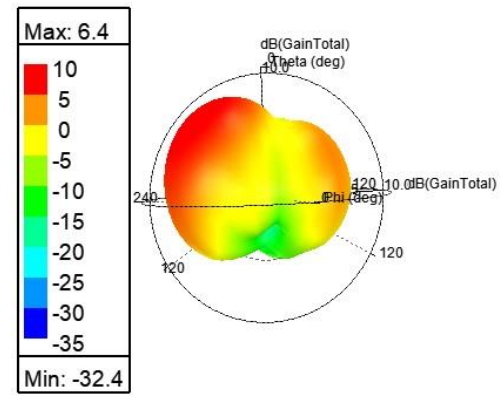

(c) Gain plot at $15.39 \mathrm{GHz}$

Fig. 11 3D polar gain plot 


\subsection{Comparative analysis of optimized Sierpinski carpet fractal antenna on FR4 substrate and} micro-machined silicon substrate

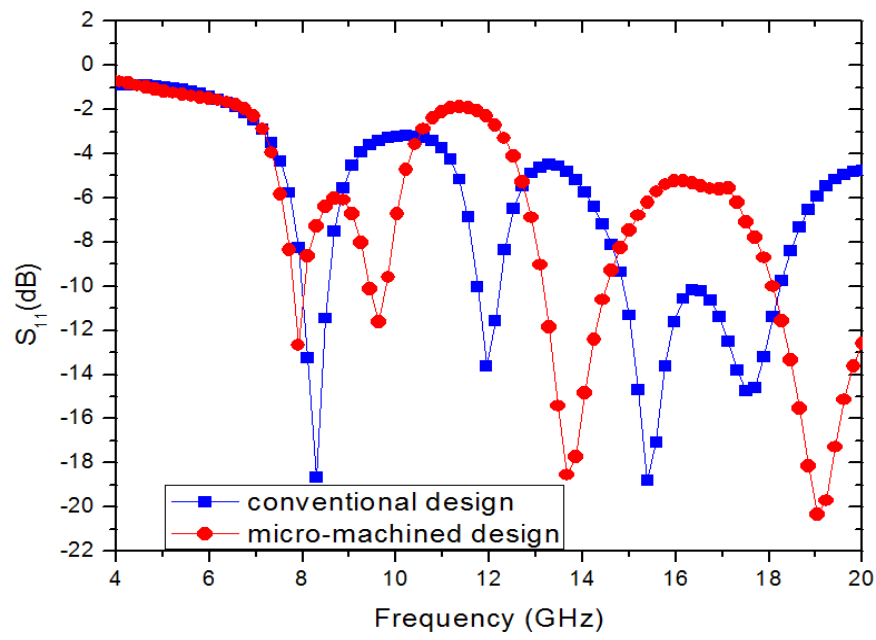

Fig. 12 Resonant characteristics of conventional and micro-machined Sierpinski carpet fractal antenna design

The resonant characteristics of the optimized Sierpinski carpet fractal antenna designed on FR4 substrate and on the micro-machined substrate have been shown in Fig. 12, which depicts the shifting in the operating frequency due to the alteration in the overall dielectric constant of the substrate material. But a considerable amount of gain improvement can be observed at a lower range of operating frequencies. A comparative analysis of various performance parameters of both the designs have been tabulated in Table 4 .

Table 4 Comparative analysis of conventional and micro-machined proposed antenna

\begin{tabular}{|c|c|c|c|}
\hline \multicolumn{4}{|c|}{ Conventional design on FR4 Substrate } \\
\hline Resonant frequency (GHz) & $\mathrm{S}_{11}(\mathrm{~dB})$ & Gain $(\mathrm{dBi})$ & VSWR \\
\hline 8.29 & -18.68 & 2.38 & 1.2634 \\
\hline 11.93 & -13.62 & 2.786 & 1.5263 \\
\hline 15.39 & -18.80 & 5.82 & 1.2593 \\
\hline \multicolumn{4}{|c|}{ Micro-Machined Patch Design } \\
\hline Resonant frequency (GHz) & $\mathrm{S}_{11}(\mathrm{~dB})$ & Gain $(\mathrm{dBi})$ & VSWR \\
\hline 7.9 & -12 & 4.4 & 1.8 \\
\hline 9.6 & -11.6 & 6.0 & 1.82 \\
\hline 13.6 & -18.5 & 5.2 & 1.26 \\
\hline 19 & -20.3 & 3.8 & 1.1 \\
\hline
\end{tabular}

\subsection{Radiation patterns of the micro-machined designs}

Radiation patterns regarding co-polarization and cross-polarization of the micro-machined version of the $3^{\text {rd }}$ iteration of the Sierpinski carpet fractal antenna design have been shown in Fig. 13. Total 8 radiation pattern, two $\left(\mathrm{phi}=0^{\circ}\right.$ and $90^{\circ}$ ) at each four operating frequencies show the required cross-polarization level at frequencies of interest in the direction perpendicular to the antenna with a maximum gain of $5.9 \mathrm{~dB}$ is observed in $\mathrm{X}$-band which is an improvement as compared to the conventional design.

Almost there is a significant improvement of $150 \%$ in the gain at X-band frequencies in the case of micro-machined design over the conventional design on FR4 substrate. The radiation patterns deteriorate at high frequencies due to radiation losses and correspondingly value of gain reduces. Moreover, 3D gain polar plots have been shown in Fig. 14. This depicts that there is broadside nature of radiation patterns at lower frequencies, but at higher frequencies, owing to losses, there are more side lobe levels in radiation patterns, and correspondingly cross-polarization level increases as shown in Fig. 12 (g) and (h). Further, a comparative analysis of the proposed design with similar fractal geometry antennas has been tabulated in Table 5, which clearly indicates that proposed design exhibits the miniaturized size with wide bandwidth as compared to the other similar designs. 


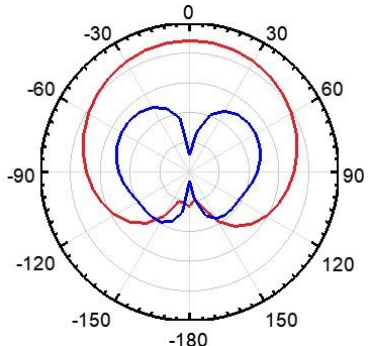

(a) $\phi=0^{\circ}$ and $\mathrm{f}=7.9 \mathrm{GHz}$

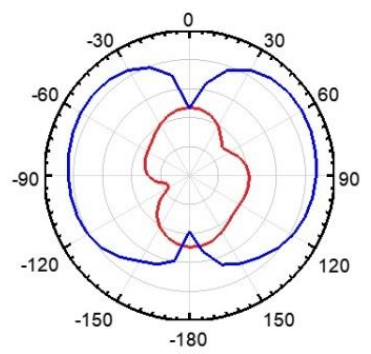

(e) $\phi=0^{\circ}$ and $\mathrm{f}=13.6 \mathrm{GHz}$

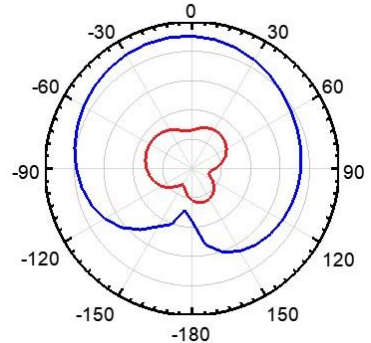

(b) $\phi=90^{\circ}$ and $\mathrm{f}=7.9 \mathrm{GHz}$

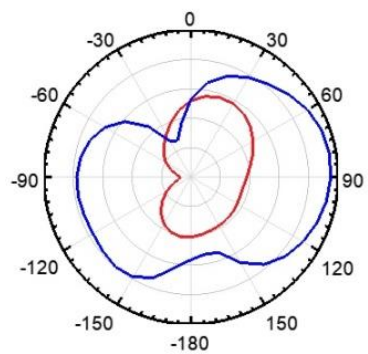

(f) $\phi=90^{\circ}$ and $\mathrm{f}=13.6 \mathrm{GHz}$

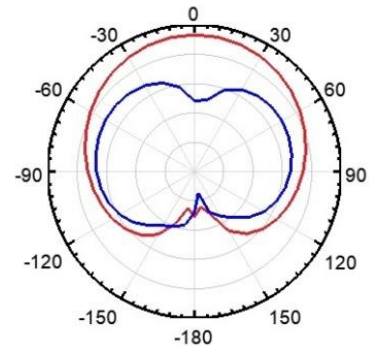

(c) $\phi=0^{\circ}$ and $\mathrm{f}=9.6 \mathrm{GHz}$

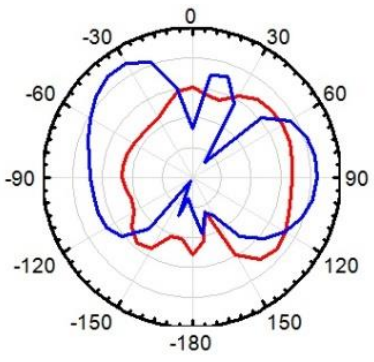

(g) $\phi=0^{\circ}$ and $\mathrm{f}=19 \mathrm{GHz}$

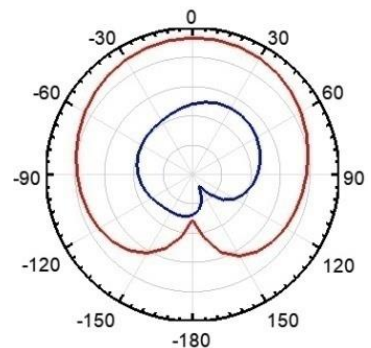

(d) $\phi=90^{\circ}$ and $\mathrm{f}=9.6 \mathrm{GHz}$

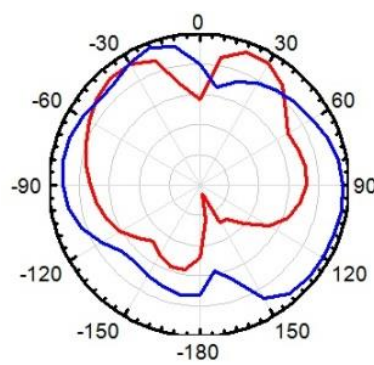

(h) $\phi=90^{\circ}$ and $\mathrm{f}=19 \mathrm{GHz}$

Fig. 13 Simulated co and cross polarization of the micro-machined antenna
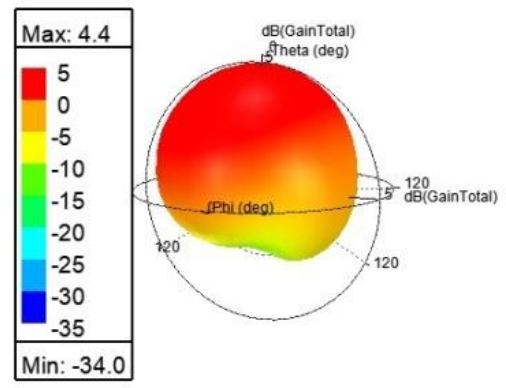

(a) $7.9 \mathrm{GHz}$

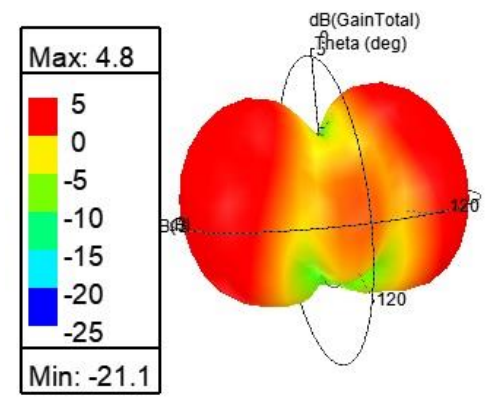

(c) $13.6 \mathrm{GHz}$
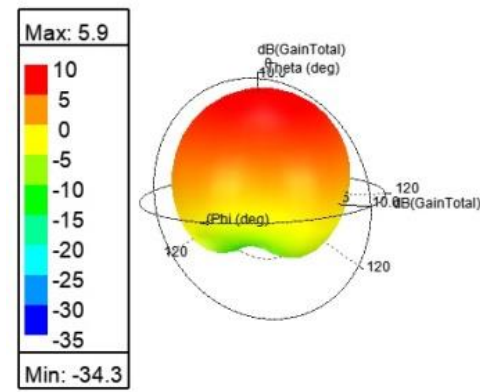

(b) $9.6 \mathrm{GHz}$
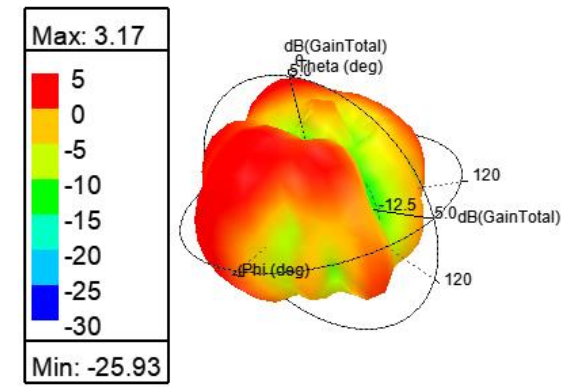

(d) $19 \mathrm{GHz}$

Fig. 14 3D polar gain plots of the micro-machined Sierpinski carpet fractal antenna

Table 5 Comparative analysis of proposed design with similar antenna designs

\begin{tabular}{|c|c|c|c|c|}
\hline $\begin{array}{c}\text { Reference } \\
\text { Papers }\end{array}$ & $\begin{array}{c}\text { Substrate } \\
\text { Used }\end{array}$ & Shape of the antenna & size $\left(\mathrm{mm}^{2}\right)$ & Frequency band (Bandwidth $(\mathrm{GHz})$ ) \\
\hline [9] & $\begin{array}{c}\text { FR4 } \\
\left(\varepsilon_{\mathrm{r}}=4.4\right)\end{array}$ & Modified Minkowski fractal & $110 \times 60$ & Multiple frequencies with very narrow band \\
\hline$[10]$ & $\begin{array}{c}\text { FR4 } \\
\left(\varepsilon_{\mathrm{r}}=4.4\right)\end{array}$ & Hybrid Fractal Antenna & $38 \times 38$ & $2.4-2.48(0.08), 5.80-5.85(0.05)$ \\
\hline [11] & $\begin{array}{c}\text { FR4 } \\
\left(\varepsilon_{\mathrm{r}}=4.4\right)\end{array}$ & Giuseppe Peano Fractal & $30 \times 30$ & $2-2.75(0.75)$ \\
\hline [12] & $\begin{array}{c}\text { Arlon } \\
\left(\varepsilon_{\mathrm{r}}=2.5\right)\end{array}$ & Sierpinski square patch & $\begin{array}{l}2052 \mathrm{~mm}^{2} \\
\text { (Area) }\end{array}$ & $1.561-1.589(0.028)$ \\
\hline [13] & $\begin{array}{c}\text { FR4 } \\
\left(\varepsilon_{\mathrm{r}}=4.4\right)\end{array}$ & $\begin{array}{l}\text { Koch fractal boundary slot } \\
\text { antenna }\end{array}$ & $100 \times 100$ & $1.825-3.375(1.55)$ \\
\hline $\begin{array}{l}\text { Proposed } \\
\text { design }\end{array}$ & $\begin{array}{c}\text { FR4 } \\
\left(\varepsilon_{\mathrm{r}}=4.4\right)\end{array}$ & Sierpinski fractal antenna & $20 \times 20$ & $\begin{array}{c}8-8.58 \mathrm{GHz}(580 \mathrm{MHz}) \\
11.7-12.17(477 \mathrm{MHz}) \\
13.3-16.9(3.3 \mathrm{GHz})\end{array}$ \\
\hline
\end{tabular}




\section{Conclusion}

An optimized Sierpinski carpet fractal antenna utilizing ant lion optimization and micro-machining process has been presented in this study. By optimizing the dimensions of the antenna using the anti-lion optimization, the required operating band with excellent impedance matching has been achieved. The ant lion optimization algorithm provides very competitive and excellent results over the other nature-inspired met heuristic algorithms because this algorithm shows high exploration and convergence speed due to boundary shrinking mechanism and elitism. The proposed design shows a resonance at 8.29 $\mathrm{GHz}, 11.93 \mathrm{GHz}$, and $15.3 \mathrm{GHz}$ with excellent impedance matching of $-26 \mathrm{~dB}$ at $8.29 \mathrm{GHz}$. The design covers the band of 8.025 GHz-8.4 GHz which further can be used for earth exploration satellite service. The maximum bandwidth of the Sierpinski fractal antenna is $3.3 \mathrm{GHz}$ in Ku-band. Further, the performance parameters of the conventional Sierpinski carpet fractal antenna have been improved in terms of gain with the process of micro-machining along with the compatibility of design with monolithic microwave integrated circuits. The maximum gain of $6 \mathrm{~dB}$ has been achieved at $9.6 \mathrm{GHz}$. There is an achievement of the significant improvement of $150 \%$ in terms of gain at X-band with the help micro-machining process.

\section{List of Acronyms}

\begin{tabular}{|c|c|}
\hline PSO & Particle Swarm Optimization \\
\hline BFO & Bacterial Foraging optimization \\
\hline ALO & Ant Lion Optimization \\
\hline VSWR & voltage standing wave ratio \\
\hline MMIC & monolithic microwave integrated circuits \\
\hline HFSS & High frequency Structure Simulator \\
\hline RL & Return Loss \\
\hline RF-MEMS & Radio Frequency Micro-Electro-Mechanical Systems \\
\hline
\end{tabular}

\section{Conflicts of Interest}

The authors declare no conflict of interest.

\section{References}

[1] C. A. Balanis, Antenna theory: analysis and design, 3rd ed. John Wiley \& Sons: New York: Wiley-Interscience, 1997.

[2] J. Anguera, A. Andujar, S. Benavente, J. Jayasinghe, and S. Kahng, "High directivity microstrip antenna with mandelbrot fractal boundary," IET Microwaves Antennas and Propagation, vol. 12, no. 4, pp. 569-575, March 2018.

[3] P. Bhartia, K. V. S. Rao, and R. S. Tomar, Millimeter-wave microstrip and printed circuit antennas, 1st ed. Boston: Artech House, 1991

[4] D. H. Warner and S. Ganguli, “An overview of fractal antenna engineering research," IEEE Antennas and Propagation Magazine, vol. 45, no. 1, pp. 38-57, February 2003.

[5] T. Y. Hsieh, J. L. Wang, and M. H. Wang, "Multi-band slot antenna," Proceedings of Engineering and Technology Innovation, vol. 4, pp. 31-33, April 2016.

[6] C. P. Baliarda, J. Romeu, R. Pous, and A. Cardama, "On the behavior of the Sierpinski multiband fractal antenna," IEEE Transactions on Antennas and Propagation, vol. 46, no. 4, pp. 517-524, April 1998.

[7] A. Kumar and A. P. Singh, "Design of micro-machined modified Sierpinski gasket fractal antenna for satellite communications," International Journal of RF and Microwave Computer-Aided Engineering, vol. 29, no. 8, pp. 1-10, April 2019.

[8] K. J. Vinoy, K. A. Jose, V. K. Varadan, and V. V. Varadan, "Hilbert curve fractal antenna: a small resonant antenna for VHF/UHF applications,” Microwave and Optical Technology Letters, vol. 29, no. 4, pp. 215-219, May 2001.

[9] A. S. Brar and J. S. Sivia, "Modified Minkowski fractal antenna for wireless applications", Journal of the Institution of Engineers (India): Series B, vol. 99, pp. 391-396, June 2018.

[10] M. Kaur and J. S. Sivia, "Minkowski, Giuseppe Peano and Koch curves based design of compact hybrid fractal antenna for biomedical applications using ANN and PSO," AEU-International Journal of Electronics and Communications, vol. 99, pp. 14-24, February 2019. 
[11] H. Oraizi and S. Hedayati, "Miniaturization of microstrip antennas by the novel application of the Giuseppe Peano fractal geometries," IEEE Transactions on Antennas and Propagation, vol. 60, no. 8, pp. 3559-3567, August 2012.

[12] I. H. Ali and R. K. Ahmed, "Fractal Sierpinski square patch antenna for GPS applications," In IOP Conference Series: Materials Science and Engineering, vol. 518, no. 4, 2019.

[13] V. V. Reddy, "Broadband Koch fractal boundary printed slot antenna for ISM band applications," Advanced Electromagnetics, vol. 7, no. 5, pp. 31-36, September 2018.

[14] A. Kumar and A. P. Singh, "Design and optimization of slotted micro-machined patch antenna using composite substrate,” Applied Computational Electromagnetic Society, vol. 34, no. 1, pp. 128-134, January 2019.

[15] A. Kumar and A. P. Singh, "Optimization of a modified rectangular patch antenna array for X-band applications," Indian Journal of Science and Technology, vol. 12, no. 16, pp. 1-18, April 2019.

[16] S. Kakkar, T. S. Kamal, and A. P. Singh, "On the design and analysis of I-shaped fractal antenna for emergency management," IETE Journal of Research, vol. 65, no. 1, pp. 1-10, January 2018.

[17] S. Rani and A. P. Singh, “A novel Design of hybrid fractal antenna using BFO,” Journal of Intelligent Fuzzy System, vol. 27, no.3, pp. 1233-1241, January 2014.

[18] I. Papapolymerou, R. F. Dryton, and L. P. B. Katehi, "Micro machined patch antenna," IEEE Transaction on Antennas and Propagation, vol. 46, no.2, pp. 275-283, February 1998.

[19] M. Zheng, Q. Chen, P. S. Hall, and V.F. Fusco, "Broadband microstrip patch antenna on micro-machined silicon substrate," Electronics Letters, vol. 34, no.1, pp. 3-4, January 1998.

[20] D. T. T. Tu, N. V. Hoc, P. D. Son, and V. V. Yem, "Design and implementation of dual band MIMO antenna with low mutual coupling using electromagnetic band dap structures for portable equipments," International Journal of Engineering and Technology Innovation, vol. 7, no. 1, pp. 48-60, January 2017.

[21] Y. D. Sergeyev, "Evaluating the exact infinitesimal values of area of Sierpinski's carpet and volume of Menger's sponge," Chaos, Solitons \& Fractals, vol. 42, no. 5, pp. 3042-3046, December 2009.

[22] F. Caldarola, "The Sierpinski curve viewed by numerical computations with infinities and infinitesimals," Applied Mathematics and Computation, vol. 318, pp. 321-328, February 2018.

[23] F. Caldarola, "The exact measures of the Sierpinski $d$-dimensional tetrahedron in connection with a Diophantine nonlinear system," Communications in Nonlinear Scienceand Numerical Simulation, vol. 63, pp. 228-238, October 2018.

[24] M. K. A. Rahim, N. Abdullah, and M. Z. A. Abdul Aziz, "Microstrip Sierpinskicarpet antenna design," Asia Pacific Conference on Applied Electromagnetics Proceedings, Johor, Malaysia, December 2005.

[25] S. Rani and A. P. Singh, "On the design and optimization of new fractal antenna using PSO," International Journal of electronics, vol. 100, no. 10, pp. 1383-1397, November 2012.

[26] S. Mirjalili, "The ant lion optimizer," Advances in Engineering Software, vol. 83, pp. 80-98, May 2015.

Copyright $\subset$ by the authors. Licensee TAETI, Taiwan. This article is an open access article distributed under the terms and conditions of the Creative Commons Attribution (CC BY-NC) license (https://creativecommons.org/licenses/by-nc/4.0/). 